\title{
Club de Mathématiques Discrètes Lyon Discrete Math Circle
}

Bodo Lass (Université de Lyon, France)

\section{History}

In 2002, Laurent Demonet, Jean-Christophe Novelli and Dimitri Zvonkine suggested that it would be nice to have the International (Russian) Tournament of Towns organised not only in Paris (and all the Russian and the many Canadian, German and Australian towns) but also in Lyon. One of the first participants in Lyon was Benjamin Scellier, a high school student living near Grenoble. He thought that we should not only have two very beautiful mathematics competitions every year but also regular mathematical club meetings - and the "Club de mathématiques discrètes"

\section{http://math.univ-lyon1.fr/ lass/club.html}

was born, quickly arriving at its actual format: two Sundays every month from 11:30 to 18:30 and three entire weeks during the holidays. The club has become attractive for more and more high school students. It started with a single student (Benjamin Scellier) and is now around 60 (15 of whom are girls) in two groups: beginners level and advanced level. Almost half of them are now from the Lyon area but most students still come from far away: Grenoble, Montpellier, Clermont-Ferrand, Dijon, Genève and, last but not least, Paris.

\section{Mathematical Instruction}

We start from high school mathematics and make excursions into all kinds of subjects. One dimensional complex (Möbius transformations) and two dimensional real projective geometries are very popular in the club. One of our favourite results is the fact that if we look at the $a \times b$ intersection points of curves of degree $a$ and $b$ and if we know that $a^{\prime} \times b$ of them lie on a curve of degree $a^{\prime}$ then the other $a$ " $\times b$ of them lie on a curve of degree $a^{\prime}, a^{\prime}+a^{\prime}=a$. In analysis, we mainly study inequalities (Jensen, Hölder, Chebyshev, Schur, Newton and Maclaurin) and the method of Lagrange multipliers, etc. In number theory, it is the Chinese remainder theorem, Hensel's lemma, Lucas' theorem, quadratic and cubic residues, primitive roots, Pell's equation, cyclotomic polynomials (their irreducibility and arithmetic properties), etc. We study functional equations and polynomials (uniqueness of decomposition into irreducible forms in one or several variables) and, last but not least, even some combinatorics: classical graph theory (theorems of König, Hall, Menger, Mader, Gallai-Milgram, etc.) and some enumerative combinatorics. A particular highlight was a Winter School in 2009, financed by the ENS Lyon, with excursions into linear programming, game theory, hyperbolic geometry and dynamical systems.

\section{Mathematical Competitions}

The aim of the club is not to prepare a competition but to do beautiful, elegant, amusing, efficient, profound, important and passionate mathematics. There are participants who do not like competitions and it is important that there is no selection in the club: every high school student who likes doing mathematics (and who wants to ask me a mathematical question per week by email) is always welcome and is free to choose their group (beginners or advanced).

Nevertheless, most participants of the club take part in mathematical competitions like the International Mathematical Olympiad (IMO) but this is based on their individual activities and totally independent of the club. Benjamin Scellier got a lot of publicity in the Grenoble area; another very talented high school student from Grenoble, Jean-François Martin, continued this and, in 2010, half of the French IMO team were students from Grenoble's International High School. The IMO is not too far away from the Tournament of Towns but the problems of the Tournament of Towns are even more beautiful, suggested by Russian mathematicians like Kontsevich, Razborov, etc. Therefore, the IMO entered naturally into the club's life. France always has to send a team of six high school students to this competition and the Lyon maths club has become more and more involved with it. In 2007, only one of the six French IMO team members (Benjamin Scellier) came from the club but he won a gold medal and $37 \%$ of the points of the French team. In 2008, the Lyon club provided three of the six team members and received $52 \%$ of the French points. In 2009 , it was four of the six team members and $70 \%$ of the points and in 2010 we had five of the six team members and $95 \%$ of the French points. Last year, all six French IMO participants came from the Lyon maths club and the French team got the best result of Western Europe (and the 14th worldwide). This Summer, however, 'only' five students from our club were selected for the French team but each of them won 17-28 points, whereas the sixth student got 11 points. Moreover, in 2015 and 2016 all the French girls selected for the EGMO (European Girls' Mathematical Olympiad) were participants of our "club de mathématiques discrètes". In particular, Lucie Wang won a gold medal.

\section{Teachers}

The most regular teachers in the club are Gabriel Dospinescu (CNRS and ENS Lyon), Theresia Eisenkölbl (Lyon University) and Bodo Lass (CNRS and Lyon University). Moreover, many others have helped: Marc Pauly, Pierre Dehornoy, Alexander Thomas, Petru 
Mironescu, Xiaolin Zeng, Nathanaël Courant, HsuehYung Lin, Jean-François Martin, Jill-Jênn Vie, Daniel El-Baz, Marko Radovanović, Antoine Michon, Matthew Fitch, Victor Vermès, Yassine Hamdi, Julien Alamelle, Christophe Barré and Christine Fenoglio.

\section{Acknowledgements}

The LABEX MILYON (ANR-10-LABX-0070) of Université de Lyon, within the programme "Investissements d'Avenir" (ANR-11-IDEX-0007) and operated by the French National Research Agency (ANR), helps disadvantaged students to participate in the club during the holidays.

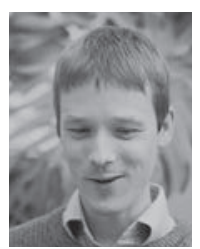

Bodo Lass studied mathematics, computer science and operations research in Bonn and Aachen. He has been enamoured with combinatorics since the German preparation of the IMOs in China and Sweden (where he won silver medals) and he loves set functions (learned from András Frank). He prepared his thesis with Eberhard Triesch and Dominique Foata and received his PhD in Strasbourg. He is now a Chargé de Recherche at CNRS. 